\title{
Application of Contactless Testing to PCBs with BGAs and Open Sockets
}

\author{
Abdelghani Renbi ${ }^{1} \cdot$ Jerker Delsing $^{1}$
}

Received: 13 September 2014 / Accepted: 13 July 2015

(C) Springer Science+Business Media New York 2015

\begin{abstract}
This paper introduces a practical test method that combines statistics with a contactless test approach. Experiments using real PCBs show the effectiveness of the method, where significant $\mathrm{z}$-scores are obtained to discriminate defective interconnects. The studied test cases involve conventional Printed Circuit Boards (PCBs) with Ball Grid Array (BGA) packages and open sockets.
\end{abstract}

Keywords Electronics testing - DfT - Contactless testing · Interconnect testing $\cdot$ PCB testing $\cdot$ Boundary scan ·

Statistical PCB testing

\section{Introduction}

A successful test strategy is key for achieving high coverage of all the possible defects, high yield and better economical testing [12]. Since long time, PCB test strategy is dominated by few test solutions only. Today, a test strategy may include the following inspections and test solutions where none of them covers $100 \%$ of possible defects.

- Manual Visual Inspection is based on human visual comparison where a reference with specific tolerances

Responsible Editor: E. J. Marinissen

Abdelghani Renbi

Abdelghani.Renbi@ltu.se

Jerker Delsing

Jerker.Delsing@1tu.se

1 Department of Computer Science, Electrical, Space Engineering, Luleå University of Technology, Luleå, Sweden is needed. It relies on trained inspectors with the help of light and magnifying glasses, the inspectors are able to recognize a defect during the PCB process steps. The inspection reports whether or not the PCB is similar to the reference. Inspection offers numerous advantages over testing as it is noninvasive and does not require any physical contacts. On the other hand with complex and dense PCBs, manual visual inspection is no longer feasible due to hidden defects which might be located under component packages such as BGA and between sides of multilayered boards.

- Automatic Optical Inspection (AOI) is an automated solution to the manual visual inspection where image processing techniques and comparison algorithms are involved. If the inspection is referential, a reference image is already stored in the processing unit to compare the image content taken by the camera. In addition to detecting defects such as shorts, opens, missing components, etc [13], AOI is exceptionally characterized by its ability of detecting wrong markings and cosmetic flaws. In the same time, for better yield, some test strategies consist of post-paste application, postplacement and post-reflow AOIs, which feedback the process for sooner correction of the process and cheaper detection of defects as the later the defect is detected the more expensive will cost. On the other hand, it is unable to detect hidden defects.

- In-Circuit Testing (ICT) which is also known as white box testing where parameters such as resistance, capacitance and others are measured with the operation of analogue components such as operational amplifier. The test is performed using dedicated test pads to ensure that each component is in its place with the correct value and thus defect-free solder joints. By assuming correct design and defect-free components, a high chance of 
correct functionality of the board is concluded. This solution requires a complete understanding of the subblocks and the components which form the the PCB as whole, thus predicting the sub-blocks responses before injecting the inputs signals [14]. ICT has several advantages as compared to the previously discussed optical solutions such as its ability to detect defective or out of tolerance components. In principal, as long as test pad are provided, ICT is able to detect hidden detects such as inner layer shorts and opens. On the other hand, ICT may not be applicable when it is not possible to afford extra physical space for test pads, due to the small feature size requirement and Electromagnetic Interference (EMI) which might be caused by the extra pads, particularly in high frequency applications. In addition, probing can be harmful to some extend especially for fragile interconnects.

- Automatic X-Ray Inspection (AXI) is a solution which has marked its birth with the emergence of BGA package. Small feature size electronics and miniaturization led to an enormous number of Input/Output (I/O) per package. BGA package was the key solution to handle this high number of $\mathrm{I} / \mathrm{O}$ interconnects inside the package. Since all the BGA solder balls are under the package, after the reflow process, it is not visible whether or not the solder balls are ensuring the expected connections to the pads. So, far, the optical solutions are unable to reveal the hidden defects. Also ICT may not be feasible when test pads are not provided due to the high frequency and small feature size requirements. This situation has marked the birth of AXI solution for PCB manufacturing chain. AXI system duty is taken place just after the soldering process to reveal defects under packages where solder joints are hidden such as BGA. AXI has another advantage over ICT, it is also used to analyze the soldering quality and thus tuning the process by looking at possible voids inside the solder joints or at insufficiently melted solder balls or at excessed or shorted in solder paste joints. All these serve long term product reliability [13]. On the other hand, AXI solution might be expensive as compared to the other test solutions from investment and test time point of view, especially when applied to every single produced PCB.

- Boundary-scan which is also known as the IEEE 1149.1 standard is a test tool since the early 1990's after the test community realized that the PCBs become physically inaccessible for probing. Boundary-scan main objective is to test interconnects without probing, thus soldering defects are tested without the need of physical access. This was realized with the support of hard- ware, basically every boundary-scan-compliant device has an architecture which supports IEEE 1149.1 standard. Boundary-scan test solution can only be applied to interconnects between digital ICs where at least one IC is standard compliant [9]. Due to the diversity of PCB components, boundary-scan test solution might not be sufficient to achieve $100 \%$ defect coverage.

- Functional Testing (FT) verifies only the functional behavior of the unit under test thus it is less effective as only limited number of signals are measured which are part of the end functionality. Most of the time, employing FT is motivated by its singular ability in detecting timing faults which cannot be revealed by ICT as it employs the proper clock frequencies during the functionality. On the other hand, FT may not encourage PCB manufacturer due to its intensive expenses and time due to the test equipment set-up and test program creation. Some times other HW needs to designed and created for interfacing the board under test with the test equipment. Moreover, some situations might occur where high troubleshooting costs for complex boards as the defective part is not known after an unexpected behavior. Also, some defects cannot be revealed by FT as they need a proper scenario for their activations, this is simply too risky for high-quality degree products [2].

Each test solution has its singular capabilities against different possible defects. Unfortunately, in some cases these test solutions may not be applicable or sufficient. This is due to the evolution of electronics towards smaller size and higher complexity. With the upcoming technology where interconnects width is below $100 \mu \mathrm{m}$, the ICT test solution is no longer feasible. On the other hand, the AXI solution is expensive and slow for testing every manufactured PCB, especially for electronics where long-term reliability is not important. Moreover, boundary-scan testing which has seen great progress from standardization point of view is not always applicable, this is due to diversity of today PCBs which may contain non-boundary-scan-compliant components $[4,11]$.

To improve the flexibility of today's test strategy and test PCB interconnects when it is not possible with today's solutions, a novel approach for testing conventional PCBs has been proposed in [11], based on broadside-coupled stripline structure where hidden interconnects are testable in a passive Unit Under Test (UUT). Figures 1 and 2 which are not drawn to scale, emphasize the most important parts. They illustrate the scenario of a separate and demountable test board that consists of a ground plane and a sensing line that is designed in such a way it overlaps with the interconnect under test. Once the test board and the UUT 


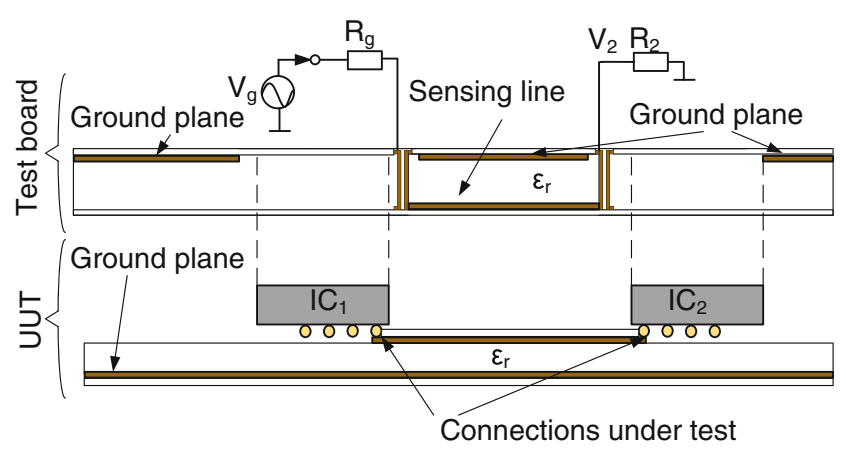

Fig. 1 Sensing line in a test board with the connection under test between two BGA packages

are closed, the sensing line and the line under test form a broadside-coupled stripline structure with high coupling through a small thickness of the solder mask layers. The voltage ratio $\frac{V_{2}}{V_{g}}$ is monitored within a frequency sweep and it is equal to half of the transmission coefficient $\frac{S 21}{2}$ when $R_{g}=R_{2}=50 \Omega$ which is the case when using a network analyzer [3]. This voltage ratio is dependent on the load capacitance of $I C_{1}$ and $I C_{2}$ as discussed in the previous work [11]. Depending on the required quality, this solution can be applied to:

- Enhance a test strategy when ICT and AOI are not applicable.

- Complement AXI solution for difficult open defect detection.

- Test hidden interconnects for BGAs when AXI is not affordable and long-term soldering reliability is not an issue.

- Improve a test strategy when boundary-scan testing is not applicable.

Contactless PCB testing which is based on broadsidecoupled stripline structure relies on the pre-known IO capacitances of the connected ICs [11]. The transmission parameter $S 21$, which is measured between the ports of the test trace in the upper part of the structure, carries informa-

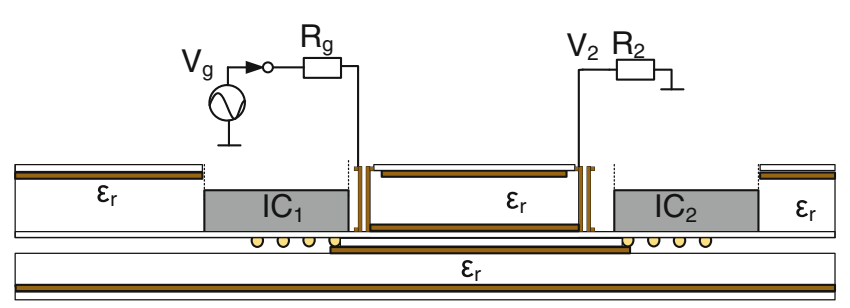

Fig. 2 Test board is mounting the UUT for testing the connections under test

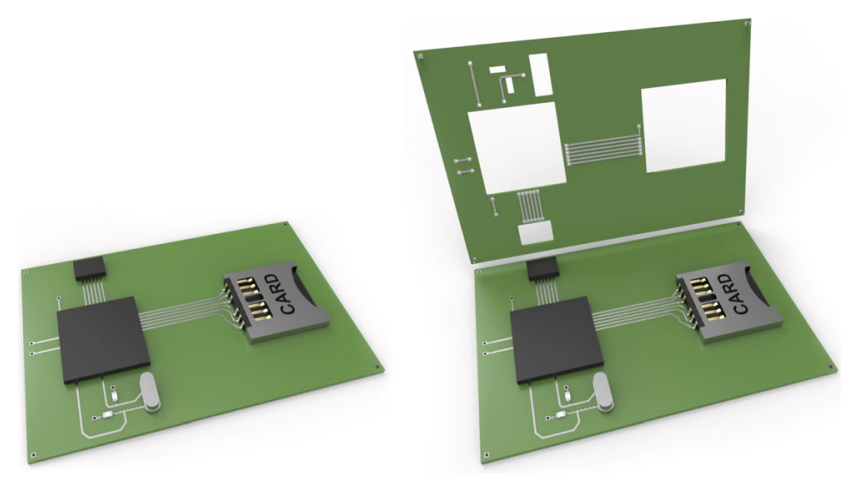

(a) UUT

(b) UUT and the test board

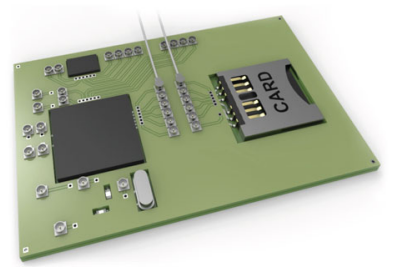

(c) Test board is mounting the UUT

Fig. 3 Testing process for conventional PCBs

tion about the terminations of the trace in the UUT which is the lower part of the structure. This information is mainly the first resonance frequency of the transmission parameter which is influenced by the ICs load capacitances. Preparing test data by simulations requires that the IO capacitances of the connected chips are well specified to determine the first resonance frequency and its variability of the transmission parameter $S 21$. In some cases, the silicon manufacturers may not provide the IO capacitances in their data sheets, simply because the IO capacitances are less important within the operating frequency from signal integrity point of views and thus limitations to simulating the first resonance frequencies for preparing test data. To keep the contactless testing approach applicable in such situations, this paper introduces the combination of the standard score statistical measurement with the contactless test approach [11]. Experiments in conventional PCBs with BGA packages and open sockets show the effectiveness of the method in detecting possible opens and bridges.

Figure 3 illustrates the 3D view of a sample board under test with its contactless test bed.

\section{Related Work}

In cases where the IO capacitances of the connected ICs are unknown or when electromagnetic simulations are not pos- 
sible, preparing test data for the contactless test approach which has been reported in [11] might not be feasible. To keep the usability and enhance the practicality of the contacltess test approach, this work demonstrates the feasibility of combining statistics with the contactless test approach for challenging inteconects in conventional PCB. Statistical test approaches have been used in several publications and mainly for chip-level testing. In [1, 7, 8, 15], the authors employed the principal component analysis (PCA) technique for defect identification and test process development while measuring the integrated circuit quiescent current $\left(I_{D D Q}\right)$ for an IC under test. Capacitive plate technique which extends boundary-scan testing for better test coverage [10] has been improved for its effectiveness by using PCA in [5]. Without PCA, capacitive plate measurements might be unreliable due to other sources of deviations such as mechanical movements [5, 6]. Generally, statistical approaches have not been yet involved heavily with PCB testing.

\section{Z-Score}

In statistics, the z-score is a quantity used to describe how much a measurement deviates from a mean of $N$ samples or a specification point for detecting outliers. In some cases, z-score is combined with Chauvenet's criterion for rejecting or accepting deviated measurements while assuming the normal distribution of the data [16]. The z-score is calculated by subtracting a sample $x$ from a target mean $\mu$ and dividing by the target standard deviation $\sigma$. Let the z-score be symbolized by $k$, the quantity $k \times \sigma$ measures the dis-

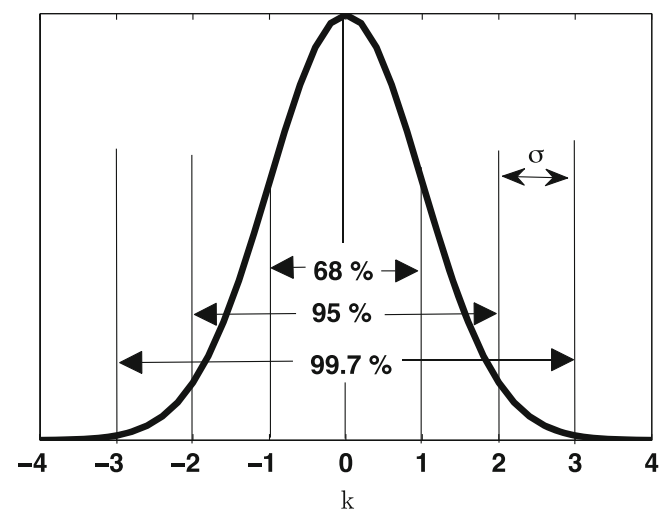

Fig. $4 k$ values for a normal distribution process tance of a sample from the target mean. Figure 4 illustrates the $k$ values of a normal distribution process.

In this work, the z-score approach is deployed by computing the number of the standard deviations in the voltage ratio $\frac{V_{2}}{V_{g}}$ within a frequency range. This ratio is measured at the sensing line to test a trace in the UUT as it is discussed in the introduction. Section 4 elaborates further, the use of z-score with the contactless test approach to detect defective traces in a UUT.

\section{Test Cases}

A design which is part of a development board has been manufactured and utilized as a UUT to demonstrate the feasibility of the approach in real use. The UUT reflects the state-of-the-art feature size, where $100 \mu \mathrm{m}$ wide interconnects, $0.45 \mathrm{~mm}$ insulator hight and $18 \mu \mathrm{m}$ thickness of the copper. The insulator dielectric constant is similar to that of FR-4, i.e., $\varepsilon_{r}=4.3$. The test bed has been manufactured using similar dimensions as the UUT, both are separated by $2 \times 25 \mu \mathrm{m}$ as solder mask thickness when they are closed for testing.

The design contains two connected BGA processors and sockets for SD card and USB peripherals. Correct and defective boards have been assembled where opens and bridges have been introduced. The pads of the biggest BGA package have been removed to ensure open defects and thus model the open termination due to probable faulty soldering process. SD card socket pins have been lifted up slightly to model soldering opens. Bridges have been introduced by

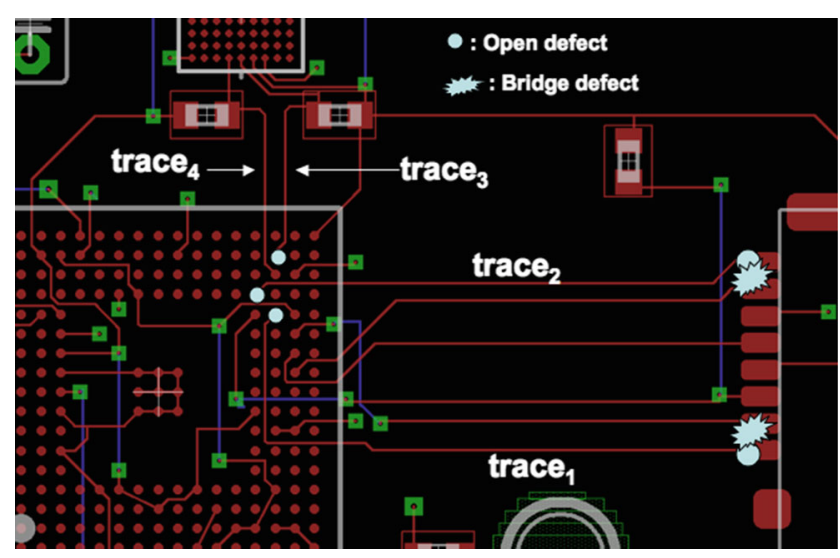

Fig. 5 Design of the UUT and locations of the introduced defects for trace 1 , trace 2 and trace 3 
Table 1 Lengths of the structure (mm)

\begin{tabular}{llll}
\hline Trace & Length & $\begin{array}{l}\text { Coupled } \\
\text { lenght }\end{array}$ & $\begin{array}{l}\text { Extension } \\
\text { ratio }\end{array}$ \\
\hline Trace $_{1}$ & 25.62 & 13.8 & 0.86 \\
Trace $_{2}$ & 20.66 & 13.75 & 0.5 \\
Trace $_{3}$ & 6.85 & 3.25 & 1 \\
Trace $_{4}$ & 8.1 & 3.3 & 1.46 \\
\hline
\end{tabular}

soldering the pin which corresponds to the trace in question to its neighbor pin. Figure 5 shows the design partially with the mapped treated defects.

Table 1 shows the important lengths of the UUT in terms of the coupled part and both extensions in each line under test. Based on the extension ratio values which are equal to $\frac{\text { Trace length-Coupled length }}{\text { Coupled length }}$, the design is made to satisfy the Design for Testability (DfT) rule to achieve $10 \%$ as the minimum margin in the difference between the first resonance frequencies of a correct and open trace as reported in [11].

The upper part of Fig. 6 illustrates the test bed with the cutouts which correspond to the surface-mounted components in the UUT. This way, the desired structure and proximity between the sensing lines and the lines under test are formed when both UUT and test bed are closed. The bottom part of Fig. 6 illustrates the UUT where the lines under test which are mentioned by Fig. 5 can be seen. Both UUT and test bed have 4 vias each, which are closed to the edges of the board, these vias are connected to the ground planes

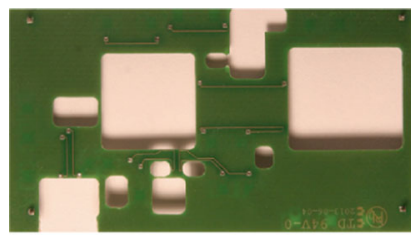

(a) Test bed (bottom side)

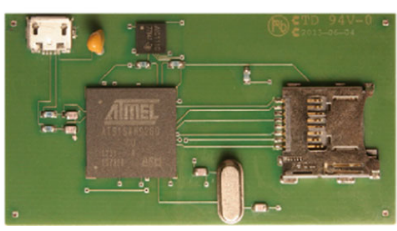

(c) UUT

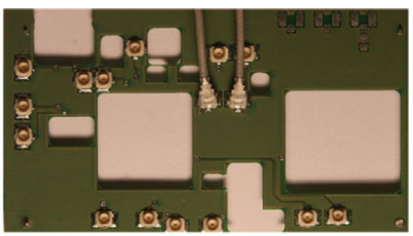

(b) Test bed (top side)

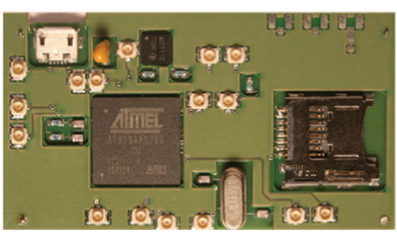

(d) Test bed mounted to the UUT
Fig. 6 Test bed and UUT and ensure the electrical connections of the ground planes as well as the mechanical alignment of both PCBs. These vias can be easily exploited by soldering pins in the test bed vias. Once the UUT and the test bed are closed with inserted pins, mechanical alignments and ground connections are realized as it is illustrated by the bottom part of Fig. 6 .

Due to the lack of information regarding the IO capacitance from chip and other components manufacturers, we may not know the first resonance frequencies of each interconnect [11]. For this main reason, we introduce a new procedure that can be applied to the contactless approach for electronics production testing. The previous simulations represent a proof that the open defects impacts are seen in the voltage ratio's first resonance frequency as well as in the level of its magnitude. This voltage ratio is equal to half of the transmission parameter $S 21$ which is measured at the sensing line. To make use of the approach without pre-simulations, one can employ a statistical approach to discriminate defective interconnects. Let $\sigma(f)$ and $\mu(f)$ are the standard deviation and the mean of the voltage ratio of each interconnect within a set of PCBs which are known to be correct. In the real production chain, the correct boards refer to those boards which have undergone extensive functional and stress testings. The variable $f$ represents the frequency. To discriminate the open defect in each interconnect in the UUT let us inspect how many $\sigma(f)$ 's are found in the difference $x(f)-\mu(f)$ in absolute value. This number is $\mathrm{k}$ where $k=\left|\frac{x(f)-\mu(f)}{\sigma(f)}\right|$. Depending on how large $k$ is, one can decide failing or passing the UUT. The value of $k$ is excepted to be important due to the deviations around the resonance frequencies. On the other hand, $\mathrm{k}$ is expected to be in the interval $[0,3]$ for normal distribution within correct PCBs.

To evaluate this test procedure the mean $\mu(f)$ and the standard deviation $\sigma(f)$ of the voltage ratio have been compiled from 10 samples population. The $\mathrm{z}$-score $\mathrm{k}$ has been calculated for each frequency for Trace 1 to $\mathrm{Trace}_{4}$ of the UUTs. Each correct trace has been tested on 5 correctly assembled boards, while each defect has been tested on 2 defective boards. The voltage ratios $\frac{V_{2}}{V_{g}}$ are obtained by measuring $\frac{S 21}{2}$.

\subsection{Trace $_{1}$ Testing}

As mapped in Fig. 5, trace $_{1}$ connects a BGA pin to an open socket. it is desired to test the ability of the contactless approach for detecting the open defect in both side of the trace. In addition, a possible bridge defect also has been tested at the socket side. 


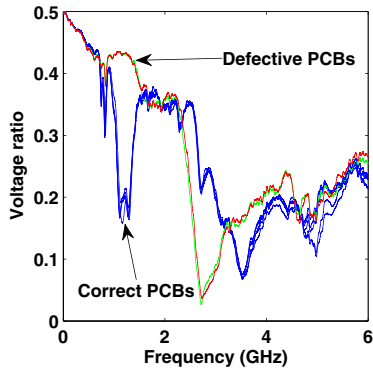

(a) Voltage ratios

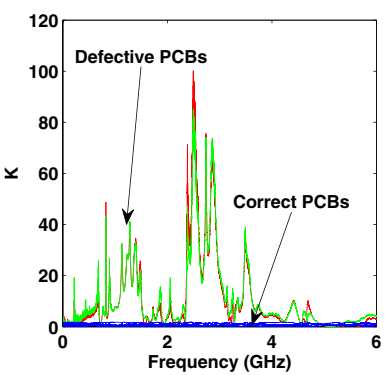

(b) Absolute values of $\mathrm{z}$ scores

Fig. 7 Open defect detection when Trace $_{1}$ is open at the BGA side

\subsubsection{Open under the BGA}

Figure $7 \mathrm{a}$ illustrates the test measurements of 5 correctly manufactured and 2 defective boards. The voltage ratios of the correct boards follow the simulated concept, although other oscillations are occurring due to the differences in the structure and crosstalk between the interconnect under test and the other solder balls. A significant margin can be achieved around the resonance frequency which is the potential source of discriminating the defect. Figure $7 \mathrm{~b}$ shows how important the quantity $k$ of the defective PCBs as compared to the correct ones.

\subsubsection{Open at the Socket}

Similarly to the open under the BGA package, Fig. 8a shows that the voltage ratios follow the simulated concept, although other oscillations are occurring due to the imperfection of the structure. It is noticeable that the plots of the 5 correct and the 2 defective boards are close to each other in similarity, this is due to the small change in the load capacitance after lifting the open socket pin. This difference is seen much better in Fig. 8b that shows the significant difference

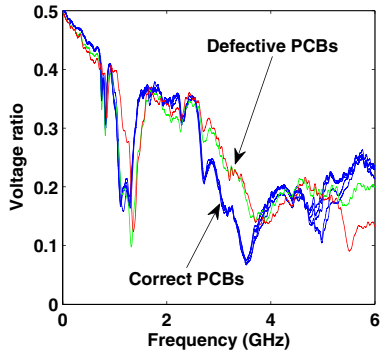

(a) Voltage ratios

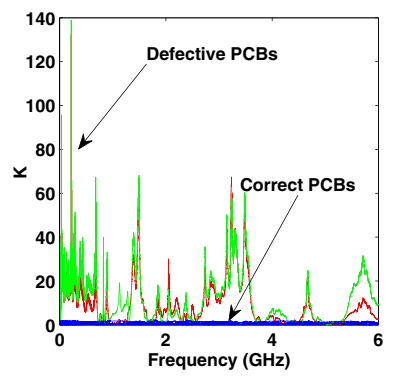

(b) Absolute values of $\mathrm{z}$ scores
Fig. 8 Open defect detection when Trace $_{1}$ is open at the socket side

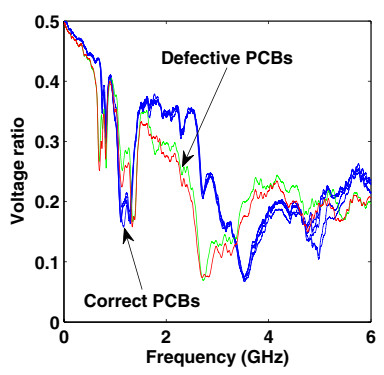

(a) Voltage ratios

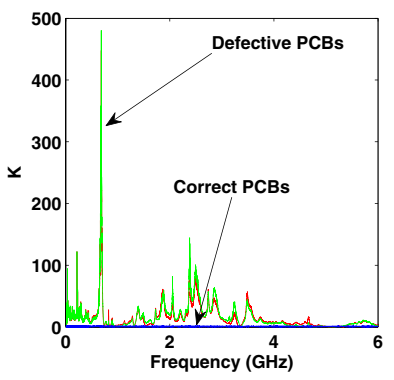

(b) Absolute values of $\mathrm{z}$ scores
Fig. 9 Bridged socket pin detection at Trace $_{1}$

between the values of $k$ of the 5 correct and 2 defective PCBs.

\subsubsection{Bridged Socket Pin}

In this case, a bridge is introduced in 2 boards between the pin in question of the socket and the neighbor pin as mapped by Fig. 5 . This bridge may occur after the reflow process due to an excess in solder paste or improper paste viscosity. According to the plots in Fig. 9a, there is a shift in the resonance frequencies of the voltage ratios that correspond to the 5 correct and 2 defective board. Based on that shift one may not decide the rejection of the defective boards. After computing the z-scores, an important difference is noticeable between the correct and defective boards in Fig. 9 b.

\subsubsection{Maximum Z-Score of Trace 1}

Table 2 collects the maximum values of z-score of each PCB test case within a $6 \mathrm{GHz}$ interval. The deviations of the correct PCBs remain acceptable, while the defective PCBs

Table 2 Trace $_{1}$ testing in different PCBs

\begin{tabular}{lll}
\hline PCB & State & Maximum $k$ value \\
\hline$P C B_{1}$ & Correct & 1.63 \\
$P C B_{2}$ & Correct & 1.78 \\
$P C B_{3}$ & Correct & 1.78 \\
$P C B_{4}$ & Correct & 1.75 \\
$P C B_{5}$ & Correct & 1.78 \\
$P C B_{6}$ & Open at BGA side & 100.08 \\
$P C B_{7}$ & Open at BGA side & 84.95 \\
$P C B_{8}$ & Open at socket side & 132.41 \\
$P C B_{9}$ & Open at socket side & 138.91 \\
$P C B_{10}$ & Bridge at socket side & 447.63 \\
$P C B_{11}$ & Bridge at socket side & 480.16 \\
\hline
\end{tabular}




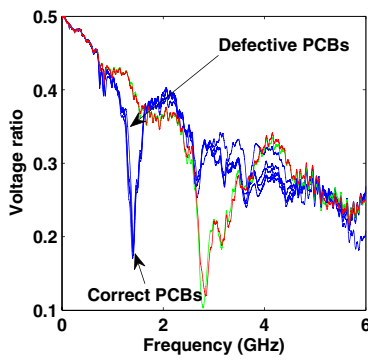

(a) Voltage ratios

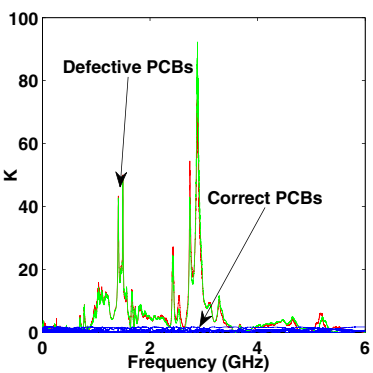

(b) Absolute values of $\mathrm{z}$ scores
Fig. 10 Open defect detection when $\operatorname{Trace}_{2}$ is open at the BGA side

can be easily discriminated as they are far from the correct boards in terms of the maximum value of $k$.

\section{2 $\mathrm{Trace}_{2}$ Testing}

Trace $_{2}$ in Fig. 5 is treated similarly to trace $_{1}$, it is desired to test the ability of the contactless approach for detecting the open defect in both sides of the trace and a possible bridge to the neighbor pin at the socket side.

\subsubsection{Open under the BGA}

The first resonance frequency of the voltage ratios in the correct and defective boards agree with the theoretical concept in Fig. 10a. One can decide easily the rejection of the defective boards. This remarkable difference has led to a significant margin in the values of $k$ as illustrated by Fig. 10b.

\subsubsection{Open at the Socket}

Similarly to the case of trace $_{1}$. The open at the socket side led to a moderate shift in the first resonance frequency of

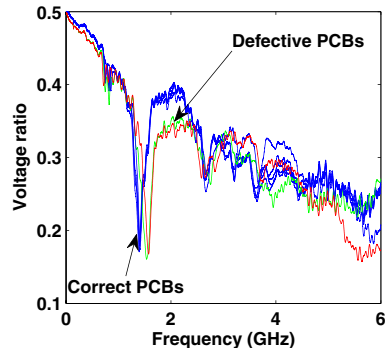

(a) Voltage ratios

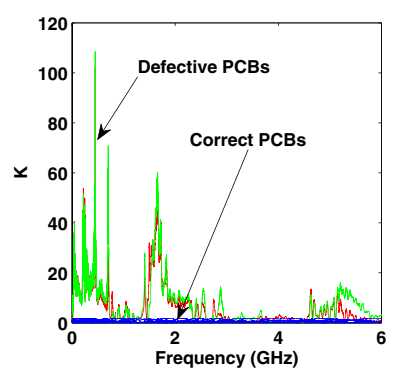

(b) Absolute values of $\mathrm{z}$ scores
Fig. 11 Open defect detection when Trace $_{2}$ is open at the socket side

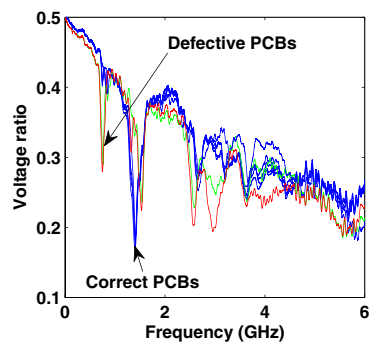

(a) Voltage ratios

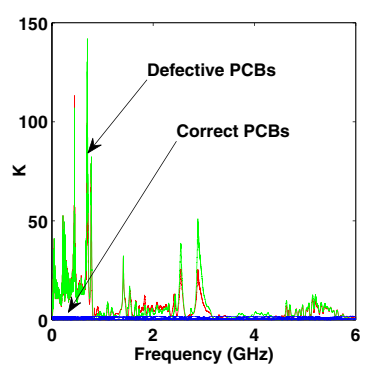

(b) Absolute values of $\mathrm{z}$ scores
Fig. 12 Bridged USB pin detection at $\mathrm{Trace}_{2}$

the voltage ratios as shown by Fig. 11a. This is due to the small change in the load capacitance after the open is created in the open socket pin. By computing the values of $k$ in Fig. $11 \mathrm{~b}$ a significant difference is noticeable between the two sets of the boards.

\subsubsection{Bridged Socket Pin}

As mapped by Fig. 5, a bridge is introduced in 2 boards between the pin in question of the socket and the neighbor pin. According to Fig. 12a, a significant difference is achieved in the voltage ratios of the two sets of the PCBs. Using the statistical procedure, the difference in the z-scores ease even further the discrimination of the defect. This is clearly seen in Fig. $12 \mathrm{~b}$.

\subsubsection{Maximum Z-Score of Trace 2}

Similarly to Trace $_{1}$, Table 3 shows that the deviations of the correct PCBs remain acceptable, while the defective PCBs

Table $3 \mathrm{Trace}_{2}$ testing in different PCBs

\begin{tabular}{lll}
\hline PCB & State & Maximum $k$ value \\
\hline$P C B_{1}$ & Correct & 1.78 \\
$P C B_{2}$ & Correct & 1.74 \\
$P C B_{3}$ & Correct & 1.72 \\
$P C B_{4}$ & Correct & 1.75 \\
$P C B_{5}$ & Correct & 1.79 \\
$P C B_{12}$ & Open at BGA side & 77.63 \\
$P C B_{13}$ & Open at BGA side & 92.24 \\
$P C B_{14}$ & Open at socket side & 101.98 \\
$P C B_{15}$ & Open at socket side & 108.53 \\
$P C B_{16}$ & Bridge at socket side & 113.22 \\
$P C B_{17}$ & Bridge at socket side & 141.97 \\
\hline
\end{tabular}




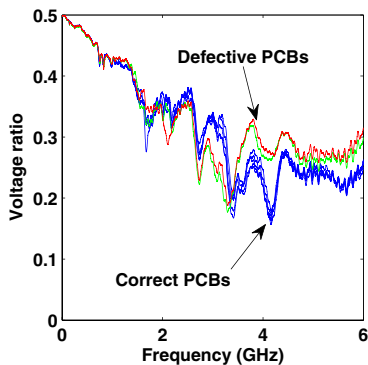

(a) Voltage ratios

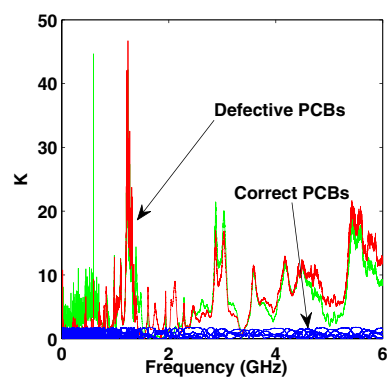

(b) Absolute values of $z-$ scores
Fig. 13 Open defect detection when Trace $_{3}$ is open at the BGA side

can be easily discriminated with important maximum value of $k$.

\section{3 $\mathrm{Trace}_{3}$ Testing}

Trace $_{3}$ which connects two BGA packages represents another test case where today's test technology may struggle to test as the small IC is non-boundary-scan-compliant. Figure 13a does not follow the simulated concept, this is expected as the coupled length is as short as $3 \mathrm{~mm}$, moreover the under test interconnect termination at the side of the small BGA is different in terms of structure and component as it contains a resistive element which was not covered in the previous work simulations. Statistically, Fig. 13b shows that the defect is detectable by the difference in the quantity $k$ between the defective PCBs and the reference ones.

\subsubsection{Maximum Z-Score of Trace 3}

Although, the coupled part of Trace $_{3}$ is too short for achieving high margin in the first resonance frequency, the maximum value of the $\mathrm{z}$-score is higher enough to reject the PCBs with open solder ball. Table 4 collects all the computed maximum values of $k$.

Table $4 \mathrm{Trace}_{3}$ testing in different PCBs

\begin{tabular}{lll}
\hline PCB & State & Maximum $k$ value \\
\hline$P C B_{1}$ & Correct & 1.77 \\
$P C B_{2}$ & Correct & 1.76 \\
$P C B_{3}$ & Correct & 1.78 \\
$P C B_{4}$ & Correct & 1.73 \\
$P C B_{5}$ & Correct & 1.79 \\
$P C B_{18}$ & Open at big BGA side & 44.65 \\
$P C B_{19}$ & Open at big BGA side & 46.7 \\
\hline
\end{tabular}

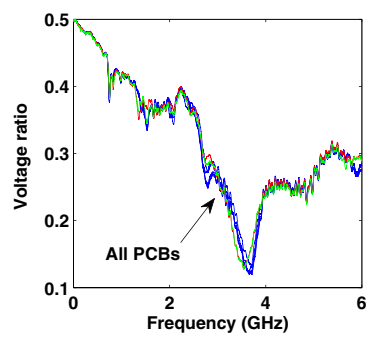

(a) Voltage ratios

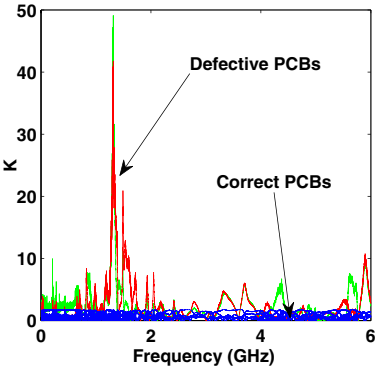

(b) Absolute values of z-scores

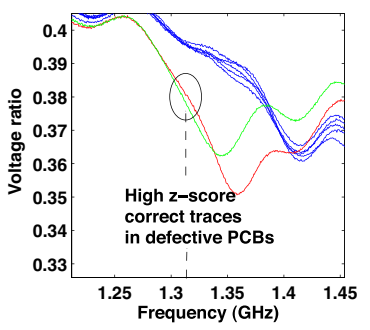

(c) Location of significant $\mathrm{z}-$ scores

Fig. 14 Correct trace testing near a defective trace

\section{4 $\mathrm{Trace}_{4}$ Testing}

As trace $_{4}$ is not defective in all PCBs, voltage ratios in Fig. 14a look similar apart from some deviations in the defective PCBs due to the other traces.

\subsubsection{Maximum Z-Score of Trace 4}

By computing the maximum value of $k$, the small scale verification is achieved and defects may show their impacts even in correct traces, especially in higher proximity cases. Looking at the plots at higher scale may not lead to a firm belief of the difference between the PCBs. On the other hand, above 40 as maximum value of $\mathrm{z}$-score will make a

Table 5 Trace $_{4}$ testing in different PCBs

\begin{tabular}{lll}
\hline PCB & State & Maximum $k$ value \\
\hline$P C B_{1}$ & Correct & 1.78 \\
$P C B_{2}$ & Correct & 1.78 \\
$P C B_{3}$ & Correct & 1.79 \\
$P C B_{4}$ & Correct & 1.77 \\
$P C B_{5}$ & Correct & 1.79 \\
$P C B_{18}$ & Close to open trace $_{3}$ & 49.1 \\
$P C B_{19}$ & Close to open trace $_{3}$ & 41.77 \\
\hline
\end{tabular}


big difference. Figure $14 \mathrm{c}$ clarifies the location of the higher $\mathrm{z}$-score which is collected in Table 5.

\section{Conclusion}

Combining the contactless test approach with the basic statistical method (z-score measurement) has been demonstrated to be feasible to conventional PCBs with BGA packages and open sockets. The experiments have shown the robustness of the approach for rejecting defective boards with high confidence. The use of the statistical method is motivated by the lack of information regarding the IO capacitance from the silicon manufacturer. Due to the high proximity in dense boards, the basic structure of the broadsidecoupled stripline might be affected by the crosstalk between the line under test and others which are close enough. For this, statistical approach is a key.

Acknowledgment The authors acknowledge the ESIS project which is supported by EU structural funds for financing this work.

\section{References}

1. Banthia A, Jayasumana A, Malaiya Y (2005) Data size reduction for clustering-based binning of ICs using principal component analysis (PCA). In: Current and defect based testing. IEEE, pp 24-30

2. Crouch A, Eide G (2006) Advances in electronic testing: challenges and methodologies. Springer, chap DFT-oriented, low-cost testers, pp 190-198

3. Hall SH (2009) Advanced signal integrity for high-speed digital designs. Wiley-IEEE Press

4. Hannu J, Häkkinen J, Voutilainen JV, Jantunen H, Moilanen M (2012) Current state of the mixed-signal test bus 1149.4. J Electron Testing - Theory Appl (JETTA) 28:857-863

5. He X, Malaiya Y, Jayasumana A, Parker K (2009) An outlier detection based approach for PCB testing. In: International test conference (ITC). IEEE, pp 1-10

6. He X, Malaiya Y, Jayasumana A, Parker K, Hird S (2010) Principal component analysis-based compensation for measurement errors due to mechanical misalignments in PCB testing. In: International test conference (ITC). IEEE, 1-10
7. Nahar A, Daasch R, Subramaniam S (2005) Burn-in reduction using principal component analysis. In: International test conference (ITC). IEEE, pp 74-81

8. O'Neill P (2008) Production multivariate outlier detection using principal components. In: International test conference (ITC). IEEE, pp 1-10

9. Parker KP (2003) The boundary-scan handbook, 3rd edn. Kluwer Academic Publishers, chap Boundary-Scan Basics and Vocabulary, pp 2-7

10. Parker KP (2012) Capacitive sensing testability in complex memory devices. In: International test conference (ITC). IEEE, pp $1-6$

11. Renbi A, Delsing J (2015) Contactless testing of circuit interconnects. J Electron Testing - Theory Appl (JETTA) 31(3):229253

12. Scheiber SF (2001a) Building a successful board-test strategy, 2nd edn. Newnes, chap what is a test strategy?, pp 1-4

13. Scheiber SF (2001b) Building a successful board-test strategy, 2nd edn. Newnes, chap inspection as test

14. Scheiber SF (2001c) Building a successful board-test strategy, 2nd edn, Newnes, chap test methods

15. Sharma A, Jayasumana A, Malaiya Y (2006) X-IDDQ: a novel defect detection technique using IDDQ data. In: VLSI test symposium. IEEE, pp 180-185

16. Taylor JR (1997) An introduction to error analysis, 2nd edn. University Science Books

Abdelghani Renbi was born in 1976 in Khouribga (Morocco), he completed his undergraduate studies in Ecole Supérieure de Technologie of Fes and in Savonia University of Applied Sciences. He received the M.Sc. in Engineering at Jönköping University in 2009. In 2012 he received the degree of Licentiate in Technology and in 2014 the Ph.D. degree, both in Industrial Electronics at Luleå University of Technology. His research interest includes design for testability for state of the art HDIs and power aware electronics. http://www.ltu.se/csee/research/ eislab.

Jerker Delsing was born in 1957 in Umeå (Sweden) and received the M.Sc. in Engineering Physics at Lund Institute of Technology in 1982. In 1985 he received the degree of Licentiate in Technology and in 1988 the Ph.D. degree, both in Electrical Measurement at the Lund Institute of Technology. Early 1995 he was appointed professor in Industrial Electronics at Luleå University of Technology where he is currently working as the scientific head of EISLAB. http://www.ltu.se/ csee/research/eislab. 